\title{
E-RESOURCES GEOGRAPHIC INFORMATION SYSTEM SYSTEM FOR MILITARY OPERATION
}

\author{
Aditya Kurniawan, Khaterini Mulyono, Mellyna and Stevan Gerardo \\ Department of Information Technology, \\ Faculty of Computer Science, Binus University, Jakarta Barat, Indonesia
}

Received 2013-06-03, Revised 2013-07-09; Accepted 2013-07-19

\begin{abstract}
Nowadays, the barometer of the strength of a country is a military force that is owned by the state. To produce a good military force in a country, it would require an easy, fast and accurate military resource management. This study utilized geographic data as a reference for the location of the logistics resources to help simplify the management of military data using spatial data in Geographic Information System (GIS). The system development approach used JSON Service technology framework and HTML 5 to improve system performance. The features development of the management of military resources associated with military operations are conducted. Results of this study generate e-resource military operation system which is swift and in accordance with the $\mathrm{W} 3 \mathrm{C}$ web standards.
\end{abstract}

Keywords: Military Operation, GIS, E-Resources, JSON Service1

\section{INTRODUCTION}

\subsection{Background}

Nowadays, the barometer of the strength of a country is a military force that is owned by the state. Military force can be seen from various perspectives such as the number of personnel, weapons systems, naval power and logistical, resources (oil production, oil consumption, oil proven reserves).

One of the military activities undertaken to carry out the mandate of Law is planning a strategy and tactics both in maintaining the sovereignty of the nation-and effort in maintaining the territorial sovereignty from foreign aggression. Due to the influence of globalization, every country military political situation faces dynamic pressure. To face these dynamic changes, the support of data and geographic information which is up to date, accurate can be obtained quickly are needed for the decision making process in determining what strategies and tactics that will be used by the military of a country. Therefore, processing and analysis of data has become a very important process in an information system Geographic Information System (GIS) is a specialized information system that manages data which have spatial information (spatial referenced) or in conclusion GIS is a computer system that has the ability to build, store, manage display geographically referenced information.

The data and information needed for military purposes such as form fields, altitude, slope, direction and distance as well as extensive, the coordinates of the place/location of the object, can be generated quickly, precisely accurately by utilizing GIS technology.

\subsection{State of the Art}

This web-based GIS military system has advantages compared to those of previous applications ever created. One of the elements of novelty of this application is that the application is able to show the information about the

Corresponding Author: Aditya Kurniawan, Department of Information Technology, Faculty of Computer Science, Binus University, Jakarta Barat, Indonesia 
coordinate information of the equipment location used by the military, such as weapons and artillery, or displays information about the location coordinates of the soldier who is in a group or battalion.

The information displayed is used to locate the exact position of military resources, so that military resources become easier to manage and well utilized. In addition, the information presented by this application can also reduce inaccuracies in the recording combat logistics.

The military software will also feature a battle management system features through a web-based GIS technology.

This study also proposes a software architecture for the new military system using JSON service oriented approach as the communication layer.

\subsection{Military Resources in Developing Country}

Based on the data of global security, the number of military personnel in developing countries is increasing by the average number between 193000-515000 personnel (GS, 2012). Based on data released by Coming Over Violence, military spending in developing countries in 1999 spent an average of $28.8 \%$ of the state budget (OCV, 2012).

\subsection{Previous Research}

\subsubsection{Military System Variability Command and Control}

Figure 1 describe software for the military command and control (C2) has component features such as Geographical Information System (GIS), tactical and planning information display with the use of military symbol that has been standardized (Ciflikli et al., 2012).

\subsection{Military System Software Architecture for C2}

According to Ciflikli et al. (2012), military system C2 can use the software architecture with publisher/subscriber design pattern mechanism on its business logics. This picture describe on Fig. 2. The C2 software architecture consists of a graphical user interface and business logic which is divided into components of subscriber and publisher data. I/O layer is responsible for managing the communication to an external system. Data Access functions to regulate the transformation of data from the database to I/O layer.

\subsection{Battle Management System for Military}

In the military operation, it is important to manage the resources used to draw up tactics in completing a given mission. Loechel et al. (2012) take on the situation and the process of the formation of the military operations of the Germany.

Military situation of Germany is divided over the situation measurement of situation awareness, after which the military leaders to plan military operations for the situation, after, a warrant was issued and executed the command and control and then provide a report. This process describe on Fig. 3. Military operations on the prototype system made by Ciflikli et al. (2012) there are features that can make military strategy maps through a static geo spatial system. This feature describe on Fig. 4. In the geo-spatial map, Ciflikli et al. (2012) provide spatial data consisting of battlefield tactic; positions of forces and enemy; and incidents report (Ciflikli et al., 2012).

According Ciflikli et al. (2012), describe on Fig. 5, the needs of the military command and control systems should focus on the needs of military strength and capabilities which can be provided by a modern software environment: military digital map; types of different reports as needed information; incorporation and evaluation report with a graphical user interface that is easy; notification features when there is a report that appears; integration of information such as images, videos and text; reports incorporation mechanism features with active communication features; feedback report feature and decision making to each expert staffs; feature where the system can be opened via mobile devices.

Military system prototype by Ciflikli et al. (2012) is proposing the military systems direction to be more on document workflow systems and content management system using the open source system that has been ready to be used. Ciflikli et al. (2012) proposed military hardware components of the system architecture and open source software as follows.

Ciflikli et al. (2012) used GeoServer as map server, Plone CMS as content management systems ZODB and PostgreSQL as the database as the security part, (Ciflikli et al., 2012) used Plugable Authentication Service (PAS) using LDAP technology as its implementation. 


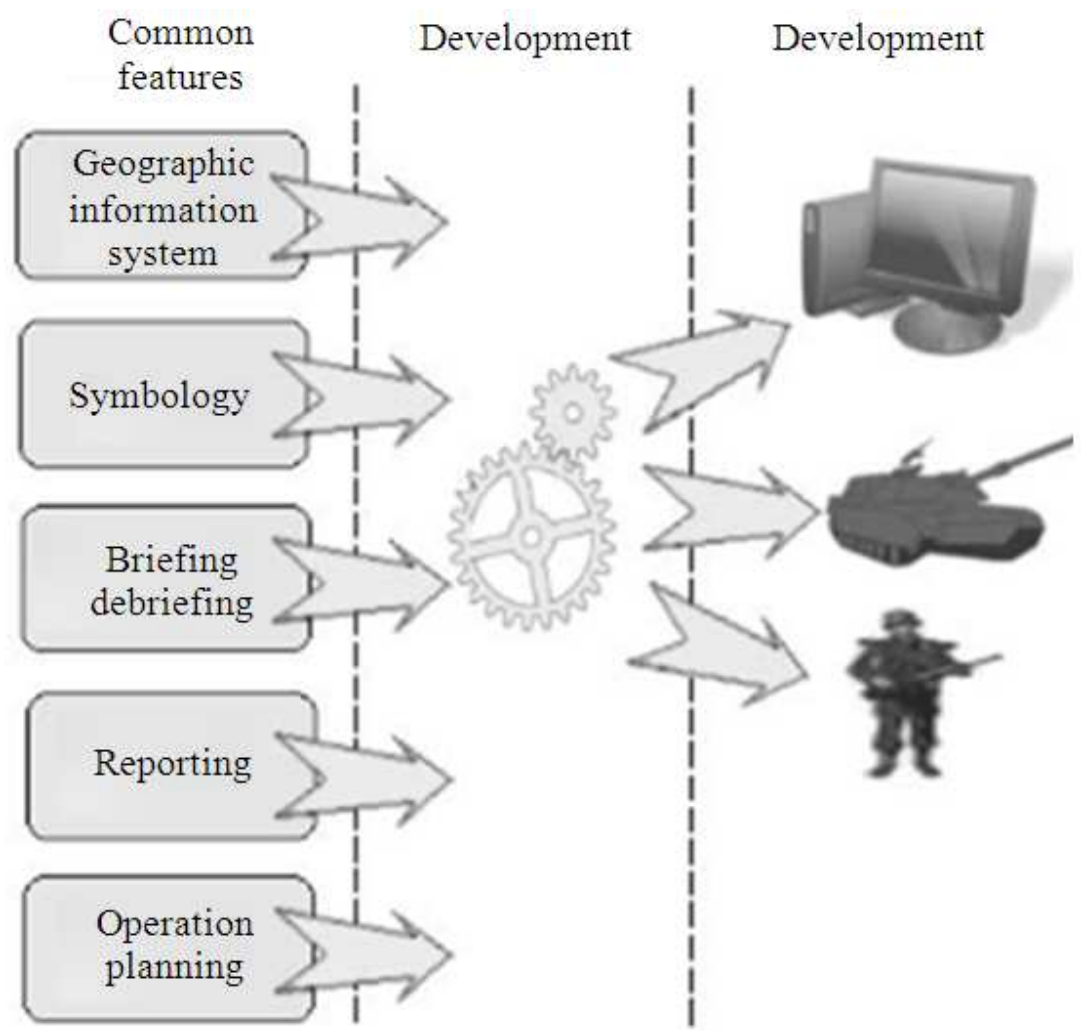

Fig. 1. Variability in command and control (Ciflikli et al., 2012)

\section{Graphical user interface}

\section{Data subscriber components}

\section{Data publisher components}

I/O layer

Data access

Fig. 2. Military system C2 software architecture (Ciflikli et al., 2012) 


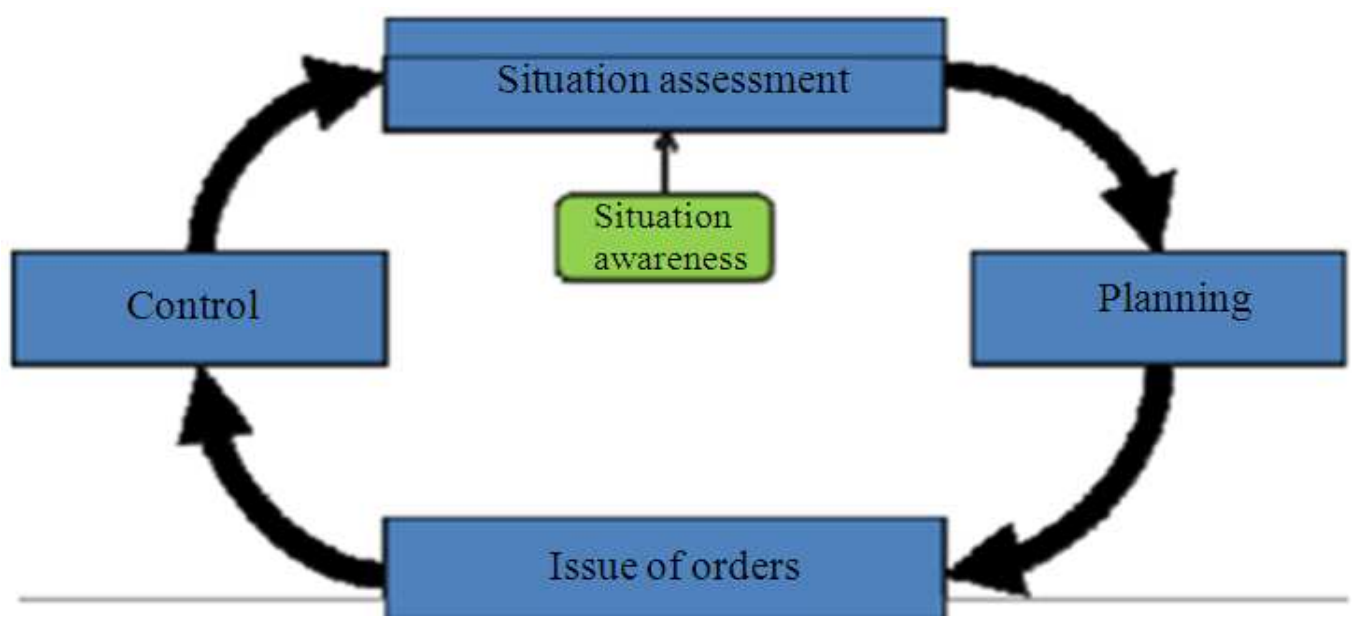

Fig. 3. Cycle of military command and control process from german (Loechel et al., 2012)

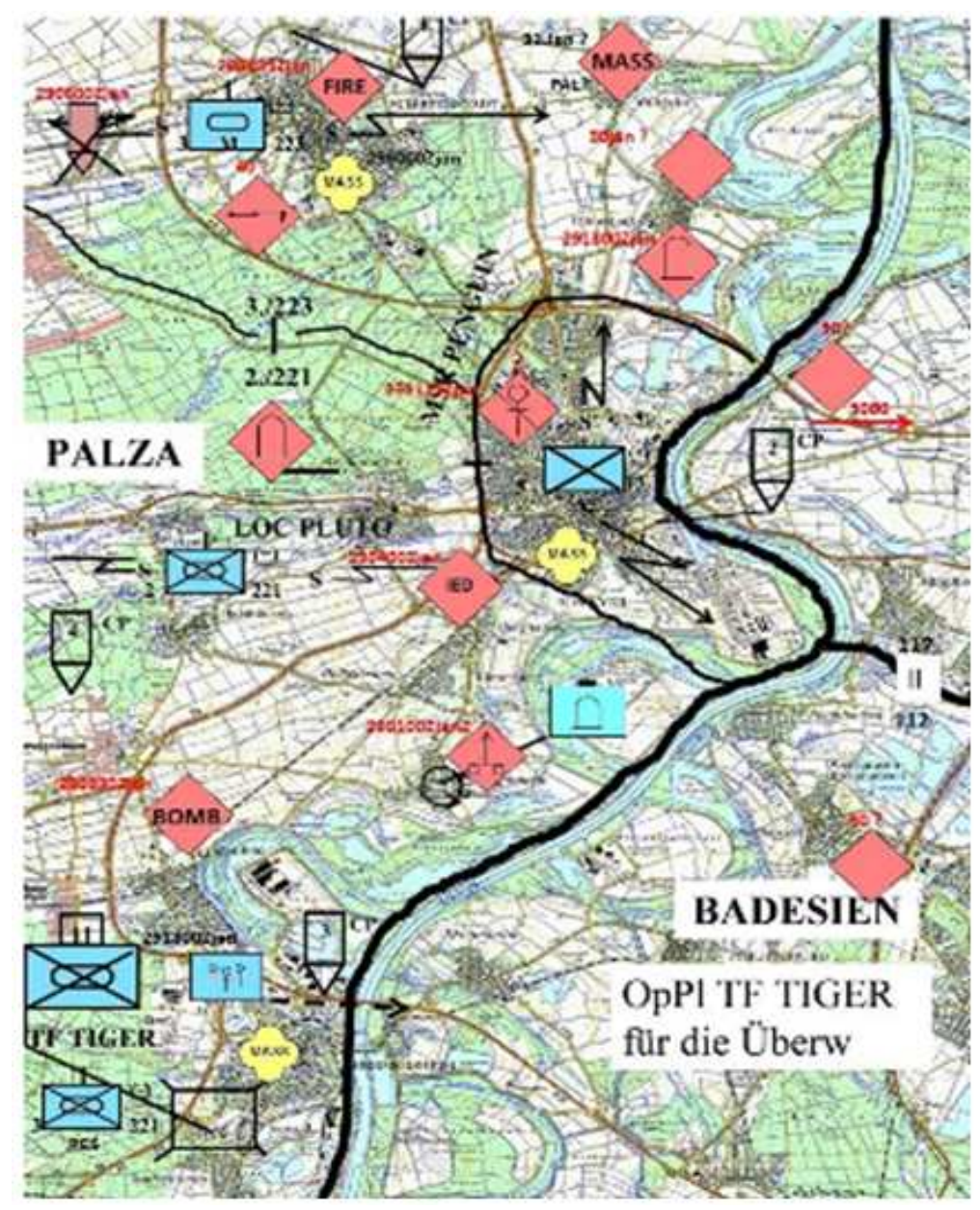

Fig. 4. Example of computer generated tactical map (Ciflikli et al., 2012) 
Aditya Kurniawan et al. / Journal of Computer Science 9 (9): 1164-1173, 2013

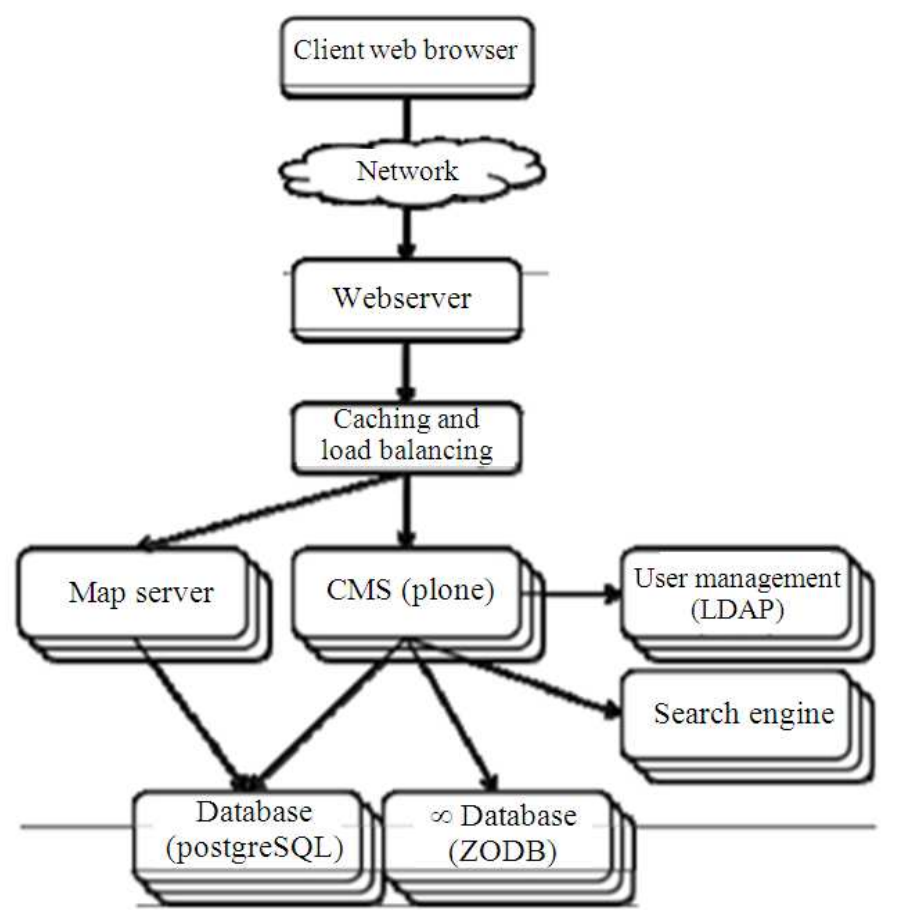

Fig. 5. Component architecture of implemented system (Ciflikli et al., 2012)

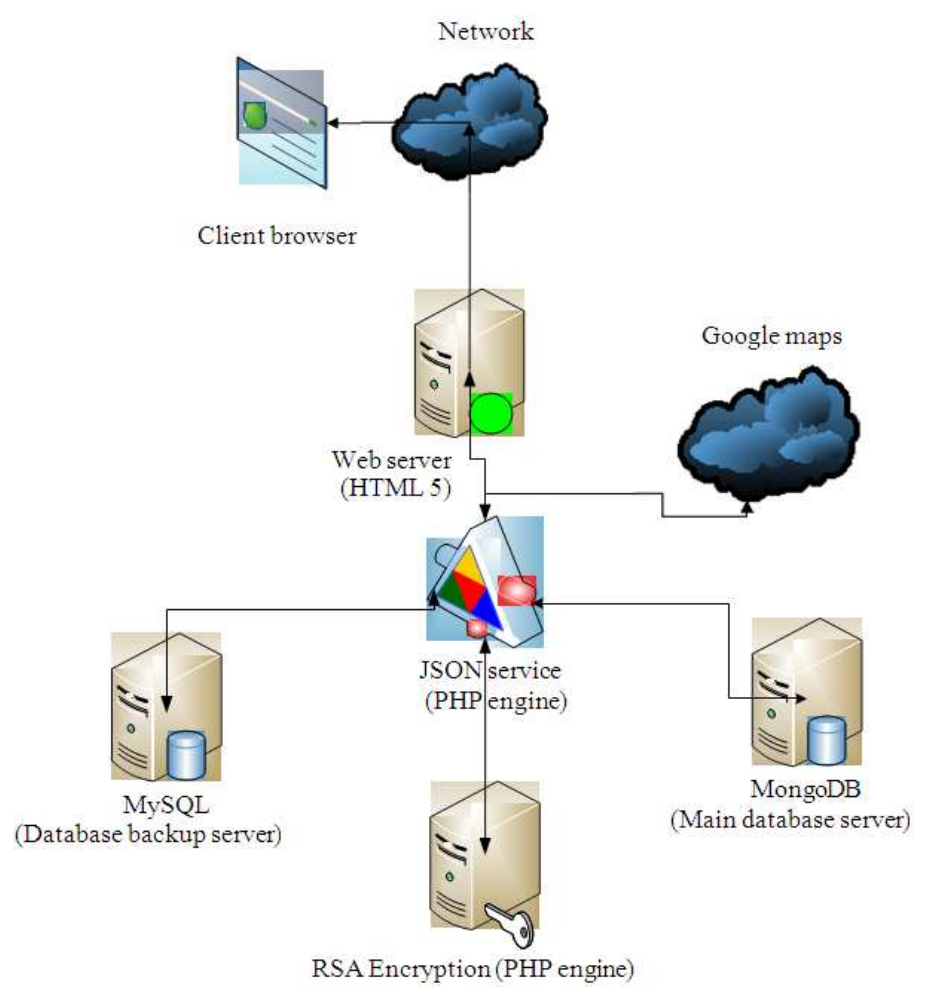

Fig. 6. Military E-resource component architecture 


\section{MATERIALS AND METHODS}

\subsection{System Design E-Resources Military}

\subsubsection{E-Resources Military System Component Architecture}

Figure 6 describe software and hardware components architecture are designed using JSON Service as the main engine of the e-resource military system. There are two main reasons for the selection of service with JSON data format: 1. Database used is using NoSQL database based BSON (Binary JSON), so that the transformation of data within its service much easier than service into the database; 2. Standardization of data service on each platform (like: Web, desktop application and m3obile device) mostly can consume JSON data.

The main database on the application uses one of the NoSQL technologies, MongoDB. While for backing up the server data we use MySQL to save the component of important military spatial information.

Since the JSON Service is a plain text and communicated through Hypertext Transfer Protocol (HTTP), it would require data protection so that important military data is encrypted. In this study, encryption used is an encryption using public key encryption algorithm of RSA public key private key using PHP technology on a separate server in order to have a maximum performance encryption of the military data.

Figure 7 explain user interface server, we use HTML 5 Javascript technology. The main reason for using this technology is the combination of these two technologies can read JSON Service which computational processes are charged to the client browser to lighten the workload of the JSON Service server.

The use of Google map as the map for geo spatial data placement is due to the sufficient completeness and updated data of Google map legend, such as roads, buildings, land and river flow data.

HTML 5 consume JSON service using JQuery javascript framework library. JQuery library aims to strike a balance between the size of the website, benefits of the features the use of a simple JavaScript framework library (Venkat, 2009).

Javascript Object Notation (JSON) is a data format serialization popular among web developers and was invented by Brendan Eich and developed by Douglas Crockford (Severance, 2012). JSON is used to encode between a servers and an AJAX application, to connect between the two servers via a web service (Severance, 2012).

\subsection{E-Resources Military Software Architecture}

In the software architecture, describe on Fig. 8, JSON Service is divided into three major sections:

- Google Map JSON Service as the processor of spatial data from Military Resources Service and Military Operation Service

- Military Operation is Used to Process the Current Military Operations Data

- Military Resources are used to process data of existing military resources and the resource transfer transactions

Data released by the JSON service should go through RSA Encryption Server so that the military data can be transferred safely through HTTP network protocol, where the private key of RSA Encryption in inside the RSA server encryption and the public key is in JSON Service server or Web Server.

\subsection{Use Case System}

Figure 9 describe military system is divided into two major feature sections, a feature for resources management and military mission operational feature.

Resource management feature focuses on the management of the use of military resources and geographical location through google map. Military resources item which are managed in this system consists of a battalion, soldiers military equipment (tanks, cannons). Battalion is a military headquarters spread across the region in a country. In addition, the items of military resources can be mobilized from one battalion to another battalion, therefore the system consider this mobilization as a transaction. Users of this feature are divided into two, regiments (regimen) and logistics.

Regimen is the group of supreme leaders of a military force, while the logistics are the military staffs who manage military resources.

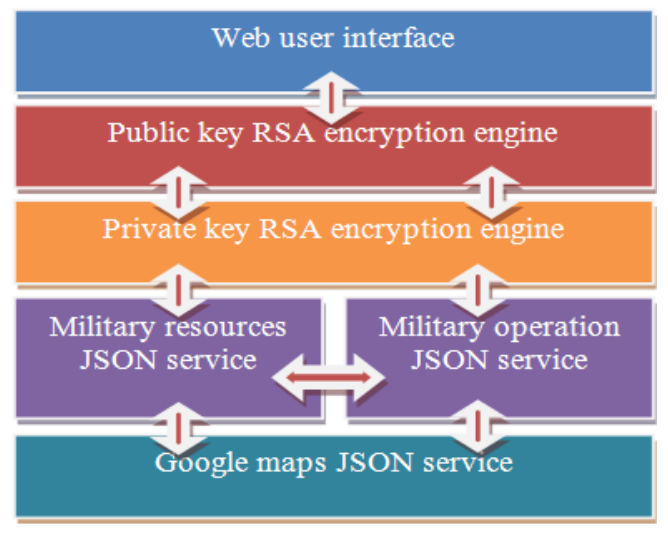

Fig. 7. Military E-resources software architecture 
Aditya Kurniawan et al. / Journal of Computer Science 9 (9): 1164-1173, 2013

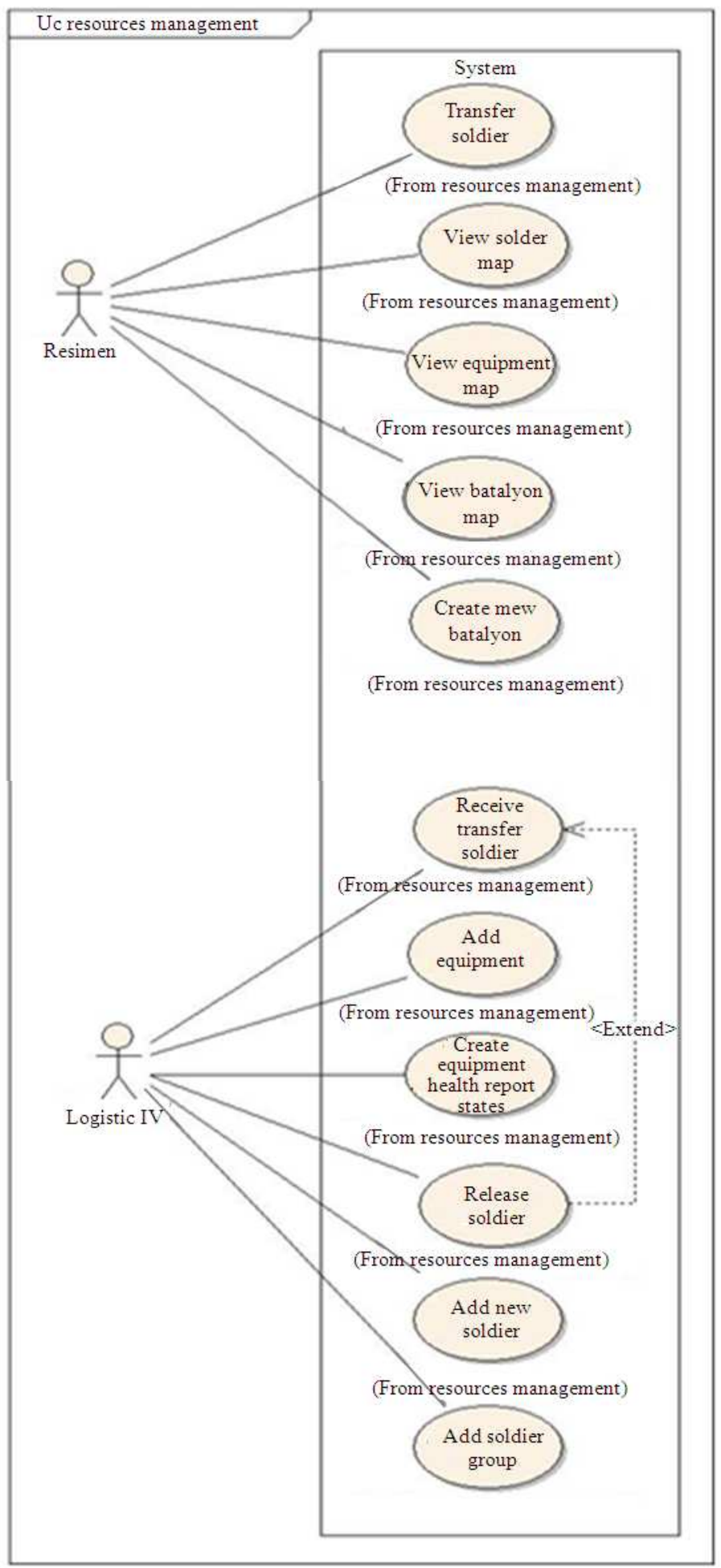

Fig. 8. Resource management use case system 


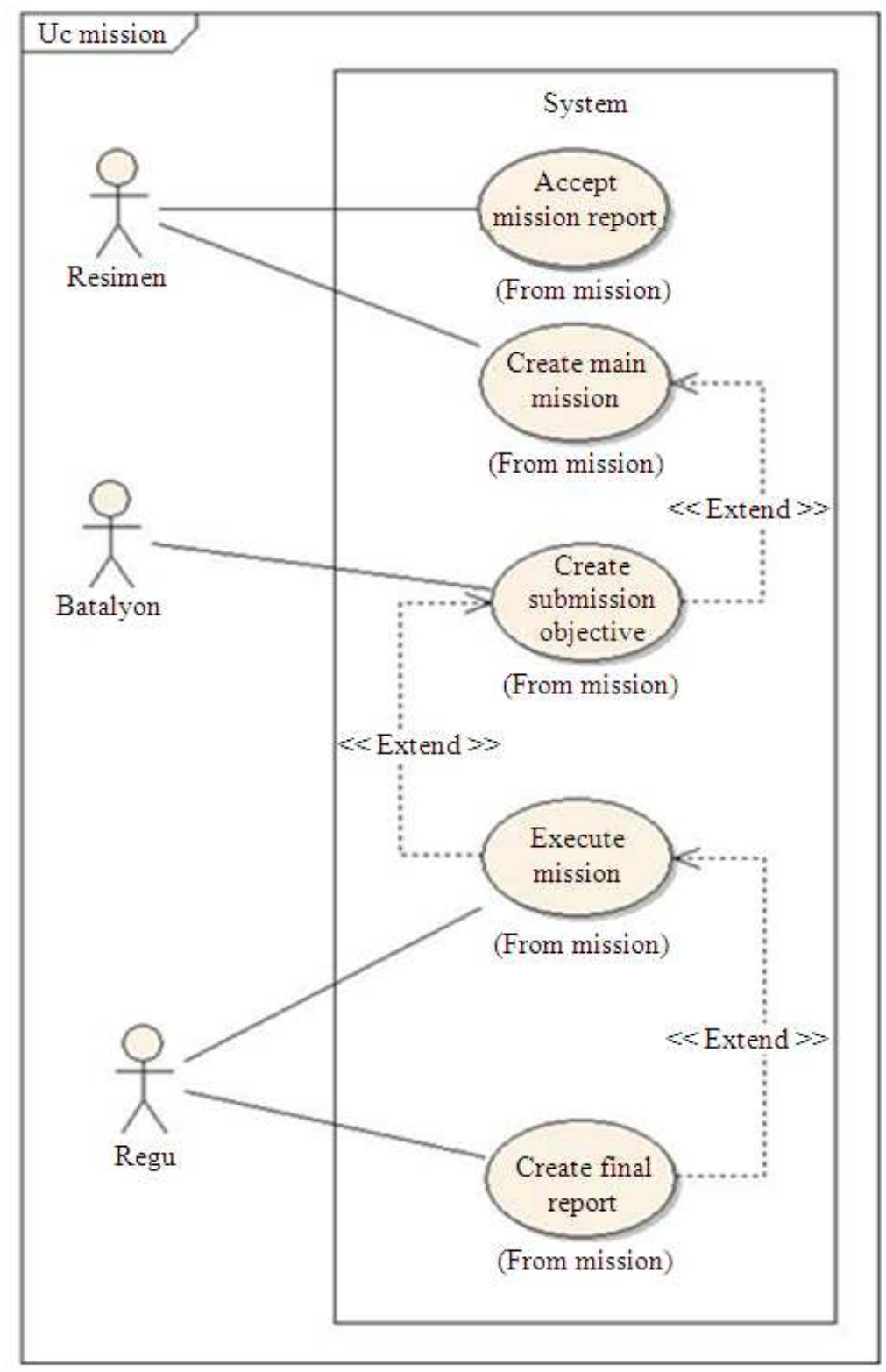

Fig. 9. Military mission operational use case system

Military mission operational feature focuses on the implementation of military operations where the military operation is taking reference from Loechel et al. (2012).

This military operations process begins in the user regimen in which the user has the right to make the primary mission of military operations. Regimen can choose which battalion would carry the mission through GIS. Then after the mission was made, then the mission of the command will be accepted by the battalion leader.
Battalion leader will choose team and team leader who will carry out the mission from the available platoon. Platoon is a group of soldiers in a battalion. Each battalion has a platoon lot. In addition, the battalion leaders can choose military equipments available to be used in missions. Battalion leaders will make a detailed mission objective and coordinate point on the Google map.

Platoon will carry out missions by fulfilling mission objectives detailed in accordance with the coordinates 
specified by the battalion leader. Once the mission is complete, the platoon leader will make a final report on the implementation of the military's mission operation for the battalion leader through the system the leader of the battalion will forward the final report to the regimen.

\section{RESULTS AND DISCUSSION}

This research has resulted in a rapid military operation e-resource based on JSON Service and HTML 5.

To measure the performance capabilities of the system, we use YSlow as a measure, because the system uses web technology. YSlow measures the ability and performance of various components of a web using various web component rules from the $\mathrm{W} 3 \mathrm{C}$ where each component is graded from $\mathrm{F}$ to $\mathrm{A}$ (YD, 2012). If the item cannot be assessed, the YSlow gives the value of n/a. The following are the measurement results.

Table 1 explan experimental results obtained, components YSlow rules that cannot be measured is the Make JavaScript and CSS External due to the JavaScript that have been encrypted using JavaScript obsufactor tool, thus making the Minify Javascript and CSS value get a grade of F. From the total assessment, this system obtained 18 rule components with grade $\mathrm{A}, 1$ rule component with grade $\mathrm{E} 3$ rule components of grade $\mathrm{F}$. Figure $\mathbf{1 0}$ is an example of mission execution feature performed by the platoon chairman using google map based on the coordinates of its mission objective.
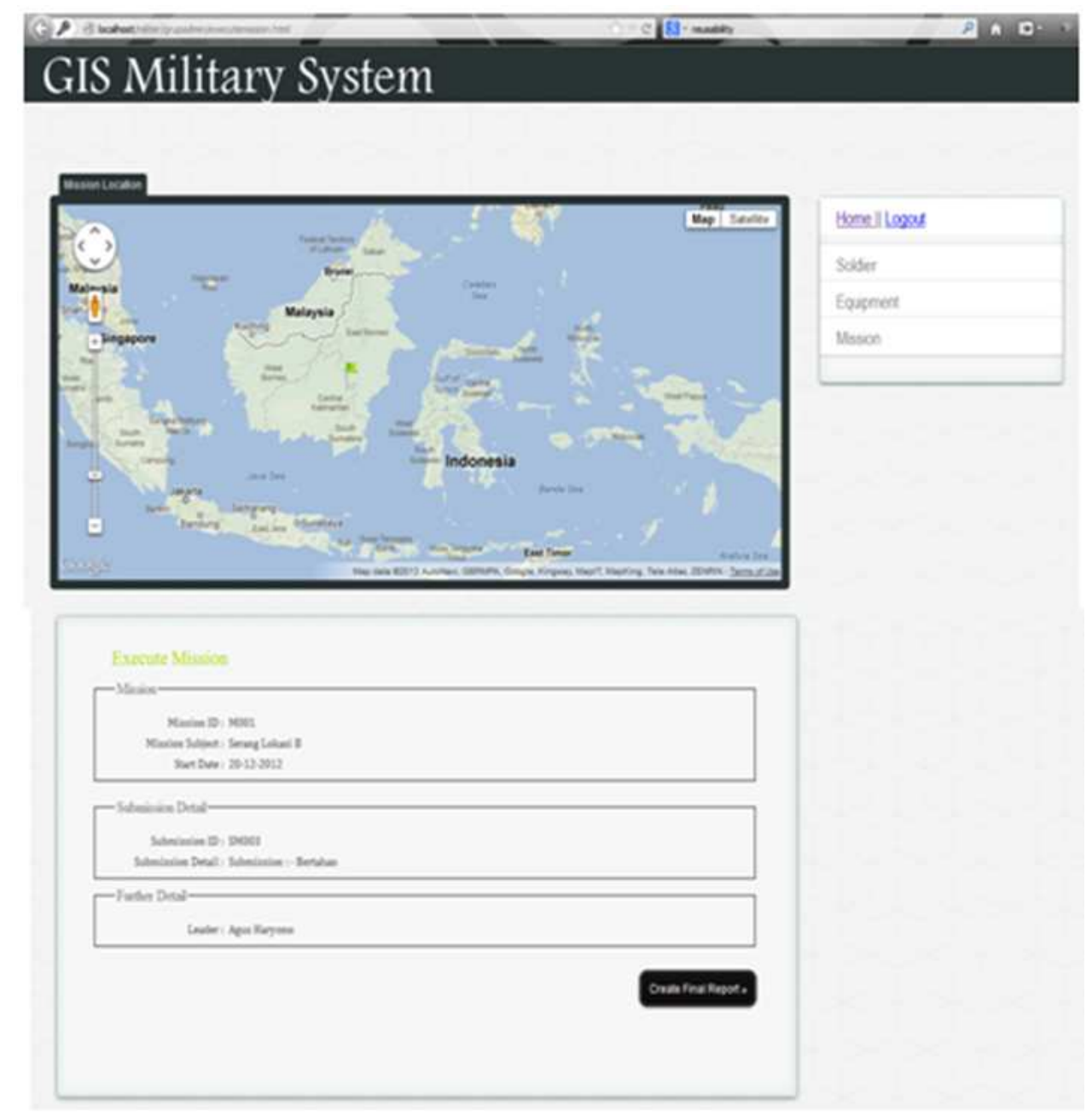

Fig. 10. Screen of execute mission feature 
Table 1. YSlow grade result

\begin{tabular}{ll}
\hline Component & Grade \\
\hline Make fewer HTTP Requests & F \\
Use Content Delivery Network (CDN) & A \\
Avoid empty src or href & A \\
Add expires headers & E \\
Compress component with gzip & A \\
Put CSS at top & A \\
Put Javascript at bottom & A \\
Avoid CSS expressions & A \\
Make javascript and CSS external & n/a \\
Reduce DNS lookups & A \\
Minify Javascript and CSS & F \\
Avoid URL redirects & A \\
Remove duplicate Javascript and CSS & A \\
Configure entity tags (Etags) & A \\
Make AJAX cacheable & A \\
Use GET for AJAX requests & A \\
Reduce the number of DOM elements & A \\
Avoid HTTP 404(not found)error & A \\
Reduce cookie size & A \\
Use cookie free domains & F \\
Avoid AlphaImageLoader filter & A \\
Do not scale images in HTML & A \\
Make favicon small and cacheable & A \\
\hline
\end{tabular}

\section{CONCLUSION}

This study discusses about the development of Military Operation e-Resource System using JSON Service technology framework and HTML 5 to improve the performance of the system.

From the results of this study found that the shortcomings of the system are due to using HTML 5 and JSON Service, thefore the HTML source code of this system can be seen in in the browser. Therefore we need an HTML Encrypter and virtual private network mechanism so that only the battalion, regimen, as well as registered military IP addresses can access the system, so civil people cannot access it

Future research development is to try to cover the shortage mentioned above by focusing on the virtual private network specifically designed for the military and Military Network application Protocol (MNP) issued by DARPA (2011) and to improve performance, the development of the system architecture component will use the distributed computing technology.

\section{REFERENCES}

Ciflikli, B., O. Ozcan, B.A. Uysal, 2012. Model driven software development for military command and control systems. Proceedings of the IEEE 36th Annual Computer Software and Applications Conference, Jul. 16-20, IEEE Xplore Press, Izmir, pp: 494-497. DOI: 10.1109/COMPSAC.2012.86

DARPA, 2011. Military Networking Protocol (MNP). Defense Advanced Research Projects Agency.

GS, 2012. Active duty uniformed troop strength. Global Security.

Loechel, A.J., G. Mihelcic and S. Pickl, 2012. An open source approach for a military situational awareness system. Proceedings of the 45th Hawaii International Conference on System Science, Jan. 47, IEEE Xplore Press, Maui, HI., pp: 1462-1471. DOI: 10.1109/HICSS.2012.109

OCV, 2010. World military expenditure.

Severance, C., 2012. Discovering javascript object notation. Computer, 45: 6-8. DOI: 10.1109/MC.2012.132

Venkat, G., 2009. Why jQuery. Vishwak Lab's White Paper.

YD, 2012. YSlow rules. Yahoo Inc.developer. 